\title{
Expert Discussion: Highlights from ABC6: Bridging the Gap and Insights in This First Virtual ABC Conference and from 10 Years ABC Consensus
}

\author{
Rachel Wuerstlein ${ }^{c}$ Fatima Cardoso $^{a}$ Renate Haidinger ${ }^{b}$ \\ a Breast Unit, Champalimaud Clinical Center, Lisbon, Portugal; ${ }^{b}$ German Breast Cancer Association, \\ Hohenbrunn, Germany; ${ }^{\mathrm{C} B r e a s t}$ Center and CCC Munich, Department of Obstetrics and Gynaecology, \\ LMU University Hospital Munich, Munich, Germany
}

The International Consensus Conference for Advanced Breast Cancer (ABC) was held virtually from November 4 to 6,2022 and renewed the international consensus guidelines for locally advanced breast cancer (LABC) and metastatic breast cancer (MBC); the panel consisted of 46 experts from 24 countries all around the world.

Over the last 10 years, the ABC Conference and Consensus has become a forum for sharing best clinical practices and information on the latest scientific updates in the field in an interdisciplinary and multiprofessional setting together with advancing the goal of improving outcomes for all MBC patients. Organization of the $\mathrm{ABC}$ Conference has now been taken over by the ABC Global Alliance.

This year the guidelines for treatment decision-making covered relevant topics such as definitions, oligo-metastatic disease, survivorship, biopsy of metastatic lesions, clinical trials, different subtypes, hereditary $A B C$, precision medicine, LABC, brain metastasis, symptom control, male $\mathrm{ABC}$, as well as symptomatic and palliative care. Two interactive round tables with participants gave further insights into the background of the panel voting.

The consensus followed 2 days of intensive and interactive live discussions with the international speakers of over 15 sessions with on demand presentations. As in the panel discussion, patient advocacy again played a major role to make this conference a success. This year, Eva
Schumacher-Wulf (Germany), an international patient advocate, gave an emotional keynote lecture „The fight against MBC - is this a battle I can win." This year's ABC award was given to Shirley A. Mertz (US), also sharing her personal experiences as well as the voice of patients' advocacy with about 900 participants from over 68 countries worldwide.

In 2022, the Team of the ABC Global Alliance will welcome again all participants in Lisbon for a special edition ABC 6.5 (November 11-12) and the next consensus will take place on November 9-11, 2023, again in Lisbon.

All information is available under: www.abc-lisbon. org and www.abcglobalalliance.org.

Both chairs of ABC6, Fatima Cardoso and Renate Haidinger, have been contributing in our ABC6 interview with their first insights.

\section{Wuerstlein: From $A B C 5$ to $A B C 6$ in 2 years' time: what have been the most relevant clinical changes in your opinion in $A B C / M B C$ ?}

Cardoso: After relevant changes for $\mathrm{HR}+\mathrm{MBC}$ at ABC5 (endocrine based therapies, mainly CDK 4/6), for ABC6 most changes occurred in HER2-positive and triple-negative breast cancer (TNBC), with the introduction of new treatment options into the standard of care; this includes new drugs, such as antibody-drug conjugates 
(ADCs) (e.g., trastuzumab-deruxtecan [T-Dxd] and sacituzumab govitecan), and checkpoint inhibitors (CPI) (mainly pembrolizumab), all resulting in survival benefit. There is special interest in the final data from T-Dxd in view of its huge progression-free survival (PFS) benefit shown at ESMO 2021.

Haidinger: For HR+ and HER2-positive breast cancer we now have treatments with 5-year survival data and new compounds coming up for TNBC which offer more treatment options and hopefully will improve survival data for these patients as well.

\section{Wuerstlein: The Covid pandemic was and still is one of the biggest challenges - for patients, clinicians but also research. Please give insight in what you think we managed well and where we still have relevant gaps for MBC.}

Cardoso: Obviously the COVID pandemic is having a terrible impact in $\mathrm{ABC}$ patients and their families, especially in palliative care: patients at the end of life not being able to be with their loved ones and dying alone is terrible and heartbreaking. I hope that a lesson learned can be that there must be a balance between measures to contain the pandemic and humanity: patients in the palliative setting or dying do not have the time to wait and should be allowed to be with family and friends.

Another important aspect was and still is the difficulty in having access to care and access to family doctors (who have been moved to Covid-related tasks). This has led to people having symptoms for a long time without having medical attention, with consequences like late diagnosis and treatment and therefore higher mortality rates.

With the pandemic we have learned the benefits but also the downsides of telemedicine. Not everything can be done at a distance and the human aspect is crucial in oncology: holding hands while giving bad news, for example. Having family and caregivers at the patients' side at the time point of breaking bad news is from a medical, ethical, and human standpoint of most important relevance. On the other hand, care delivered at home is also very important and improves quality of life of patients and caregivers.

For research: at the beginning we had a stop/delay in clinical trials, namely in recruitment. However, the field has quickly adapted, and some lessons must be learned for the future: using digital means can greatly facilitate a lot of steps in research, make things less heavy operatively, allowing monitoring by tele-tools, avoid all unnecessary parts, etc.

In addition, we have seen all stakeholders collaborating at an unprecedented speed to bring new treatments from basic science to clinical implementation: basic and clinical researchers, regulatory agencies, funders, sponsors, hospital administrators, politicians, and policy makers. So, if it was done for Covid, it can and should be done for cancer as well!

Virtual communication facilitated a lot of things for patients, caregivers, the medical teams, and researchers, in different areas from multidisciplinary tumor boards to virtual conferences and educational events.

Finally something very important for the ABC Global Alliance fight: we have seen that flexibility at work is not only possible but also productive (home working, flexible hours). This is something we have been advocating for $\mathrm{ABC}$ patients for a long time and we hope that now changes in work-related issues can be implemented in all countries.

Haidinger: In many countries by now there are additional ways (e.g., digital solutions) to still support patients and keep them on their medication. Even though there are still changes of treatments, delays, and also stopping of treatments. As numbers of infected Covid-19 patients rise, early detection and surgeries might be delayed and will lead to worse outcomes. Randomized clinical trials (RCTs) are more difficult to conduct.

We will still try to improve survival of patients and also their quality of life.

\section{Wuerstlein: ABC Conferences and Consensus have become a worldwide respected vote to bring MBC forward. What is the most relevant success over the past 2 years since $A B C 5$ and what would you like to achieve between $A B C 6$ and 7 ?}

Cardoso: The ABC Global Alliance has established the priority goals/objectives for the field (ABC Global Charter) and the first one is to double the median overall survival (OS). When we started our work, in the developing world, the median OS was 2-3 years. We finally see that substantially changed for 2 of the 3 breast cancer subtypes: HER2-positive and HR+, which now have a median OS of about 5 years. Unfortunately, this is not true all over the world, but only in countries where access to new therapies exist. This is an ongoing fight and another goal in the Charter.

What we now need to see is also the substantial improvement in OS for triple-negative $\mathrm{ABC}$ as well. I hope we will be able to see it in time for ABC7.

My vision for the conference itself is to have the whole breast cancer field united, collaborating, sharing knowledge and resources. Together we are much stronger. It was with great sadness that we saw ESMO leaving the $\mathrm{ABC}$ community and we can only wish and hope that ESMO returns to work with all of us, for the benefit of ABC patients. Fortunately, we continue to have the full 
support and collaboration of many other organizations such as ESO, ASCO, AGO, St. Paul-Nice French guidelines, NCCN, Eusoma, EONS, SIS, ESTRO, European Cancer Organization, and the 180 members of the $\mathrm{ABC}$ Global Alliance worldwide, which include numerous patient and professional organizations. We should all be together and work together, around the same goals, for the benefit of the patients. This is the only way to defeat this disease.

Haidinger: We will still try to improve survival of patients and also their quality of life. We will focus to pursue the goals of the ABC Global Alliance charter and work on more support for patient advocates and patients all over the world, especially for the less developed countries.

\section{Wuerstlein: We learnt since $\mathbf{2 0 2 0}$ to handle virtual conferences in a more and more professional way for organizers and participants. What is your feedback of ABC6 (virtual) and what is the idea behind the invitation to ABC6.5 in Lisbon next year already?}

Cardoso: Obviously, virtual conferences are not the same as face-to-face ones, especially for a special conference as $\mathrm{ABC}$, where networking and sharing experiences is an essential component. Therefore we have decided to have a special edition of $\mathrm{ABC}$ next year that we have called ABC6.5. This way we can all be together again, hopefully!

However, there are also advantages of having a virtual conference: people who would otherwise not be able to travel and participate, can easily join online and have access to presentations, discussions, and guidelines.

The future for $\mathrm{ABC}$ might be a mix: have the face-toface meeting but record the live sessions and discussions and make them accessible on demand.

Haidinger: While we all have done a lot of virtual meetings and conferences and they basically worked, we definitely miss meeting in person and discussing, networking, and socializing. As the ABC Conferences are so special with patient advocates involved from the beginning, ABC6.5 in 2022 is even more important.

\section{Wuerstlein: At this ABC Conference, patient advocacy sessions were a major input. What is your most relevant take-home message from these sessions?}

Cardoso: In these sessions, we usually discuss important topics not covered in other sessions of the conference that are relevant for patients and healthcare professionals. This year we have focused on:

1 The role of big data and artificial intelligence for $A B C$ management. It is not the future but already the pres- ent! In many different areas such as pathology, PROMs, delivery of systemic therapy, etc.

2 Onco-brain: what is it, what can be done, what kind of research do we need?

3 Mental health for both patients and healthcare professionals.

4 Inequalities in access to clinical trials: difficulties around the world to guarantee the same access to trials and new drugs, specific cultural barriers, communication with regulatories and sponsors, bigger involvement of patients and advocates in the design of clinical trials including the choice of the best endpoints.

Haidinger: In the patient advocacy sessions we complemented the main conference and touched many very important aspects for $\mathrm{MBC}$ patients, e.g., how to manage stress, anxiety, depression, and resilience, if and how big data and artificial intelligence can help MBC patients, different aspects of onco-brain, which is a common reality, and we dove deeply in the inequalities in access to clinical trials and tried to find solutions and asked that independently of race, gender, and ethnicity patients should be involved in RCTs

\section{Wuerstlein: LABC still is a very relevant topic worldwide. What are the most important messages from the Consensus for this?}

Cardoso: We needed to revise this topic since the previous guidelines were several years old. In addition, because of the pandemic we are seeing an increase in LABC worldwide, due to delay of diagnosis and access to treatments. In the context of $\mathrm{ABC}$, we define $\mathrm{LABC}$ as inoperable extensive local disease, without distant metastases. This clinical entity is usually "lost" because it is neither early nor metastatic breast cancer. And it is often excluded from clinical trials, especially inflammatory locally advanced disease. This entity is very frequent in low-middle income countries and trials and guidelines are really needed.

While updating the guidelines, we have included the new targeted drugs such as CDK 4/6 inhibitors, checkpoint inhibitors and PARP inhibitors for these patients, as well as the best chemotherapy options for the different subtypes. One of the remaining open questions is related to the duration of systemic therapy, especially after local therapies.

Our mission is also to identify where data gaps exist and fight for clinical trials to be run to generate those data.

Haidinger: $\mathrm{LABC}$ remains a problem in all countries, especially those with low income. But also in Europe there was a trend to larger tumors, in the Covid pandemic, but also in general.

Mainly the addition of guidelines for inclusion of new therapies into this setting is important for these patients. 


\section{Wuerstlein: Regarding the Consensus at ABC6: any vote you were surprised about, and any result of panel vote you disagree with?}

Cardoso: There was only one recommendation where a high consensus was not obtained: about the role of anthracyclines in HER2+ LABC disease. I am afraid that, when voting took place, the panel may have forgotten we were talking about inoperable locally advanced, extensive disease and not the usual neoadjuvant approach for large operable early breast cancer. In fact, after our live discussion, we asked the audience to vote and $70 \%$ voted in favor of including anthracyclines in this situation, which is what I strongly believe as well.

Haidinger: I am always surprised by the differences in treatment and management in other parts in the world, which show the importance of these $\mathrm{ABC}$ guidelines for advanced and metastatic breast cancer.

\section{Wuerstlein: What is the key message from the Consensus votes on Saturday in your opinion?}

Cardoso: ABC6 was a quite straight-forward and easy consensus, which obviously makes me happy because this is an indication that the level of evidence for each recommendation is increasing. In $\mathrm{ABC} 1$, most recommendations had the lowest level of evidence, which is "expert opinion." Ten years later, in ABC6, most recommendations have either level 1 or 2 of evidence. This means we have better quality in research, better endpoints (trials focusing on survival and not just on PFS) and overall this is an extraordinary advancement for $\mathrm{ABC}$ patients.

Haidinger: We are getting closer and closer to personalized and precise treatments, but we need to ensure that they are available for patients, no matter where they come from, which race, gender, or ethnicity they belong to and how much money they have. There is still a lot of work to do from all of us on these topics.

\section{Wuerstlein: Both of you are active members of the ABC Global Alliance - please give a short description of what this network is useful for and how you are going to further develop this initiative? What is the link between the ABC Global Alliance and the ABC Conferences (or vice versa)?}

Cardoso: The ABC Global Alliance was born out of the $\mathrm{ABC}$ Conference. When we created the ABC Conference we realized that there was a real need for a united community. The movement generated around the conference led to the creation of a global alliance that puts together all the stakeholders - researchers, healthcare professionals, patients, advocates, pharma and diagnostic companies, policy makers, etc. - from all over the world, different perspectives, working together, around the same goals. The first action of the ABC Global Alliance was the definition of the ABC Global Charter (https://www.abcglobalalliance.org/abc-global-charter/), which is a comprehensive needs assessment and defines priorities for the $\mathrm{ABC}$ field. All our projects and actions tackle at least one of these 10 goals.

The ABC Guidelines are a fundamental tool for work of the ABC Global Alliance to improve survival, by applying best treatment based on evidence by a multidisciplinary specialized team.

One of our major objectives is to foster and help the adaptation and adoption of the $\mathrm{ABC}$ Guidelines around the world, through what we call "Best of ABC at..." There were already several successful events for example in Latin America and New Zealand.

Haidinger: The ABC Global Alliance, which was founded by Fatima Cardoso, and where I am the Director of the General Assembly, aims to improve lives of patients with advanced and metastatic breast cancer all over the world. One of our main goals was to double survival by 2025 and we are still working to achieve this goal. This alliance brings together everyone who wants to contribute to our goals and we develop programs for patient advocates as well as for patients, do campaigns (e.g., right now: "The truth about working with advanced breast cancer"), and develop many other projects.

\section{Wuerstlein: What is the most relevant information from $A B C 6$ you will take into account to change your practice or attitude after the $A B C 6$ Conference?}

Cardoso: Particularly useful for my clinical practice were the discussion and guidance around biopsies of the metastatic sites and what to do if biomarkers differ in the primary and metastatic tumors. Also very important were the discussion and guidelines for the management of brain metastases and the relevance of drug interactions between new targeted therapies and commonly used therapies such as gastric protectors, vitamins, and antibiotics. This will be especially important with the new selective estrogen receptor degraders (SERDs) and both healthcare professionals and patients must be aware of this problem.

Haidinger: I am not treating patients. If I were: chemotherapy alone is not the main option in treating MBC patients and more testing has to be done to find out where it is indicated. 
Wuerstlein: Let's speculate about the $\mathbf{2}$ years to come - what do you think will be the most important difference in $M B C$ in 2 years time at $A B C 7$ ?

Cardoso: We need more advances in TNBC. How can we apply in practice the different subgroups within TNBC, how to best treat each of them, and how to improve survival of these patients? I hope that in 2 years we will see important advancements in these questions.

Haidinger: I still hope that we might see some patients that might be cured of MBC, but certainly we will see more prolonged survival.

\section{Wuerstlein: Underserved patient populations have also been discussed at $A B C 6$. What is the definition of this population in Europe, and what is the most relevant vote of $A B C 6$ for this population?}

Cardoso: The issue of inequalities is very real in $\mathrm{Eu}-$ rope, not only between countries but also very much within each country. More and more we as oncologists need to adapt our decisions to the health coverage of the patient and that is heartbreaking and horrible for the patient and family.

The are underserved populations in Europe, such as those in lower socioeconomic, less educated, rural areas. And a growing group are the migrants. Often they do not even have access to basic medical care, much less specialized care, such as oncology. We need to continuously work on the communication and fight against these inequalities.

Haidinger: The underserved patient populations have to be more included in RCTs.

\section{Wuerstlein: Side effect management coming along with drug development becomes more and more important. There were several votes at the Consensus in this area, please conclude from your point of view the most important take-home message from this chapter.}

Cardoso: In addition to what I already discussed, I would like to highlight here new side effects, such as management of interstitial lung disease (ILD). New targeted agents bring new side effects and need for research, safety management, and education.

Haidinger: The more precise treatments we have, the more well-known side effects occur that now are somehow different to manage and new side effects evolve that have to be closely monitored. It means that the treating physicians or first contact persons for patients need to be better educated about them and how to handle them.

\section{Wuerstlein: Is there anything you would like to add?}

Cardoso, Haidinger: We invite you all to listen to the wonderful lectures given by Eva Schumacher-Wulf and Shirley A. Merz during the ABC6 Opening Ceremony, sharing their experiences and efforts.

We would also like to highlight the memorial video done in memory of 3 extraordinary and inspiring women who we have lost in these 2 years: Karen Benn, Marie Pandeloglou, Bella Kaufman. We have also lost many important people in the breast cancer community and they were also remembered in the opening session: José Baselga, Angelo Di Leo, Gouri Bhattachharrya, and in Portugal Noémia Afonso. And many, many more around the world: since ABC5 we have lost 1.2 million people, mostly women, to advanced breast cancer. It is for them we have to continue the $\mathrm{ABC}$ work!

We thank both chairs of the ABC6 conference for their first insights into this year's ABC6.

\section{Conflict of Interest Statement}

Fatima Cardoso: Personal financial interest in form of consultancy role for: Amgen, Astellas/Medivation, AstraZeneca, Celgene, Daiichi-Sankyo, Debiopharm, Eisai, GE Oncology, Genentech, Gilead, GlaxoSmithKline, Iqvia, Macrogenics, Medscape, MerckSharp, Merus BV, Mylan, Mundipharma, Novartis, Pfizer, PierreFabre, prIME Oncology, Roche, Sanofi, Samsung Bioepis, Seagen, Teva, Touchime. Institutional financial support for clinical trials from: Amgen, Astra-Zeneca, Boehringer-Ingelheim, Bristol-Myers-Squibb, Bayer, Daiichi, Eisai, Fresenius GmbH, Genentech, GlaxoSmithKline, Ipsen, Incyte, Nektar Therapeutics, Nerviano, Novartis, Macrogenics, Medigene, MedImmune, Merck, Millenium, Pfizer, Pierre-Fabre, Roche, Sanofi-Aventis, Sonus, Taiho Oncology, Tesaro, Tigris, Wilex, Wyeth. Non-financial disclosures: President ABC Global Alliance and ABC Consensus Conference and Guidelines. Member/Committee Member of ESMO, ESO, EORTC-BCG, IBCSG, SOLTI, ASCO, AACR, EACR, SIS, ASPIC.

Renate Haidinger: No conflict of interest to declare.

Rachel Wuerstlein: Served as advisor, consultant, speaker and travel grant: Agendia, Amgen, Aristo, Astra Zeneca, Boeringer Ingelheim, Carl Zeiss, Celgene, Daiichi-Sankyo, Eisai, Exact Sciences, Genomic Health, Gilead, Glaxo Smith Kline, Hexal, Lilly, Medstrom Medical, MSD, Mundipharma, Mylan, Nanostring, Novartis, Odonate, Paxman, Palleos, Pfizer, Pierre Fabre, PumaBiotechnolgogy, Riemser, Roche, Sandoz/Hexal, Sanofi Genzyme, Seattle Genetics /Seagen, Tesaro Bio, Teva, Veracyte, Viatris; FOMF, Aurikamed, Clinsol, Pomme Med

\author{
Chair \\ Rachel Wuerstlein, MD \\ Breast Center and CCC Munich \\ Department of Obstetrics and Gynaecology \\ LMU University Hospital Munich \\ Marchioninistr. 11 \\ 81377 Munich, Germany \\ Rachel.wuerstlein@med.uni-muenchen.de
}




\section{Participants}

Fatima Cardoso, MD

Director Breast Unit Champalimaud Clinical Center and President ABC Global Alliance

Av. De Brasília s/n

1400-038 Lisbon, Portugal

fatimacardoso@fundacaochampalimaud.pt
Renate Haidinger

Director of the General Assembly of the ABC Global Alliance President Brustkrebs Deutschland e.V. (German Breast Cancer Association)

Lise-Meitner-Str. 7

85662 Hohenbrunn, Germany

renate.haidinger@brustkrebsdeutschland.de 